\title{
Longitudinal analysis of somatic development in paediatric patients with IDDM: genetic influences on height and weight
}

\author{
R. W. Holl, E. Heinze, M. Seifert, M. Grabert, W. M. Teller \\ Department of Paediatrics I, University Children's Hospital, Ulm, Germany
}

Summary Normal growth and development, as well as the prevention of overweight, are major goals in the treatment of paediatric patients with insulin-dependent diabetes mellitus (IDDM). We therefore evaluated longitudinally the anthropometric measurements of height and weight, as well as bone age, in an unselected group of 389 patients with IDDM treated at one institution. In order to identify genetic influences on these parameters, height and weight were determined in 186 unaffected siblings and 177 pairs of parents. At diagnosis, patients were slightly taller than average (median $\mathrm{z}$ score: +0.37 ). During the subsequent course of diabetes, age-adjusted heights decreased progressively for the first 9 years, catching up again after more than 10 years of diabetes. Bone ages were progressively retarded with increasing duration of diabetes. In 76 patients of 18 years or older, median $\mathrm{z}$-score for height was +0.30 , not different from their unaffected siblings (median z-score: +0.22 ). The correlation with midparental height was identical for diabetic and nondiabetic siblings $(r=0.43)$. In contrast, children with diabetes were significantly heavier (z-score for weight: +0.74 compared to +0.34 in unaffected siblings; $p<0.002$ ). Obesity developed primarily during and after puberty. We conclude that: 1) during the course of diabetes, longitudinal growth is temporarily reduced and maturation is delayed in children with diabetes compared to unaffected siblings. However, this effect of diabetes is transient and small compared to genetic influences on height in an individual child. 2) As a group, children with IDDM become significantly overweight, which is likely to increase the cardiovascular risk during adulthood [Diabetologia (1994) 37: 925-929].

Key words Height, weight, obesity, siblings, genetic factors, bone age.
Several authors have reported reduced longitudinal growth in children and adolescents with insufficiently controlled IDDM $[1,2]$. In recent years, based on more efficient insulin treatment, short stature appears to be less of a problem, while patients with long-

Received: 1 December 1993

and in final revised form: 6 April 1994

Corresponding author: Dr. R.W.Holl, Universitätskinderklinik, Prittwitzstrasse 43, Box 3880, D-89070 Ulm, Germany

Abbreviations: BA, Bone age; $\mathrm{CA}$, chronological age; $\mathrm{GH}$, growth hormone; IGF-I, insulin-like growth factor I; NPH, neutral protamin Hagedorn-insulin; SDS, standard deviation score ( $z$ score); IDDM, insulin-dependent diabetes mellitus; BMI, body mass index. standing diabetes have been repeatedly recognised as overweight [3-5].

We previously published a prospective multi-centre study, comparing diabetic children to a control group of 102 healthy children. In 49 patients followed-up for at least 3 years after the onset of diabetes, reduced height together with increased weight was shown [3]. The present study is based on a much larger, unselected group of patients treated at a single institution, encompassing the entire paediatric age range from early childhood to young adults. It has been repeatedly hypothesized that rapidly growing, tall children bear an increased risk for IDDM. Unfortunately, previous studies were often hampered by using unsuitable reference populations [6-8]. In addition to the individual genetic background, regional differences 
and secular changes both in height as well as tempo of growth and maturation, have to be considered.

In order to discriminate between genetic and metabolic influences on stature in diabetic children, unaffected first-degree relatives provide an ideal control population, which has rarely been evaluated previously [9]. Therefore, the aim of our study was to longitudinally follow-up height, weight and body mass index in an unselected group of paediatric patients with IDDM, treated at a single centre, and relate these data to the respective measurements in a group of nondiabetic siblings and parents.

\section{Patients and methods}

We evaluated 389 patients with IDDM (201 girls, 188 boys), who had been treated and followed up at our institution. The majority of data was collected between 1980 and $1992(92.4 \%)$. Of a total of 421 patient-records available, 32 patients were excluded from analysis for the following reasons: one or two parents of non-German descent $(n=25)$, coexistence of celiac disease $(n=6)$ or long-term steroid replacement therapy for adreno-genital syndrome $(n=1)$. All patients were treated by $2-4$ daily injections of a free mixture of regular and NPH insulin. Both in-patient as well as out-patient care was provided by the same group of doctors, nurses and dieticians. The therapeutic regimen used at our centre and the degree of metabolic control achieved have been published recently $[10,11]$.

Height was determined by a Harpenden stadiometer up to 1989 and subsequently by a newly-developed electronic stadiometer with automatic recalibration (Fa. Busse, Ulm, Germany; inter-observer coefficient of variation: $0.1 \%$ ). Bone ages were determined from an X ray of the left hand and wrist according to Greulich and Pyle [12]. All parents and siblings were asked to participate in this study. Families were included only if both parents were non-diabetic and available for measurement; similarly, diabetic siblings were excluded. Complete data were available for 177 pairs of parents and 186 unaffected siblings. In addition to the information described in this paper, blood pressure was recorded in all subjects.

\section{Statistical analysis}

As height and weight are dependent on both age and sex, z scores (SD scores) were calculated based on the data from the Zurich longitudinal growth study ([13]; data for BMI were kindly supplied by A.Prader). Among the anthropometric studies published so far, these data provide a suitable comparison for height and weight in children from our region. In order to eliminate a possible bias due to variation in the number of examinations per year, all measurements from yearly intervals, based either on chronological age or on duration of diabetes, were averaged for each patient. Data were collected using a custom-designed computer program previously developed at our institution [10]. Relevant data were transferred to a spreadsheet program (Lotus Symphony). For statistical evaluation, non-parametric tests were applied (Wilcoxon-test, Spearman's coefficient of correlation; SAS software package). Group data were expressed as median $\left[25^{\text {th }}, 75^{\text {th }}\right.$ percentiles], statistical significance was assumed for $p<0.05$.

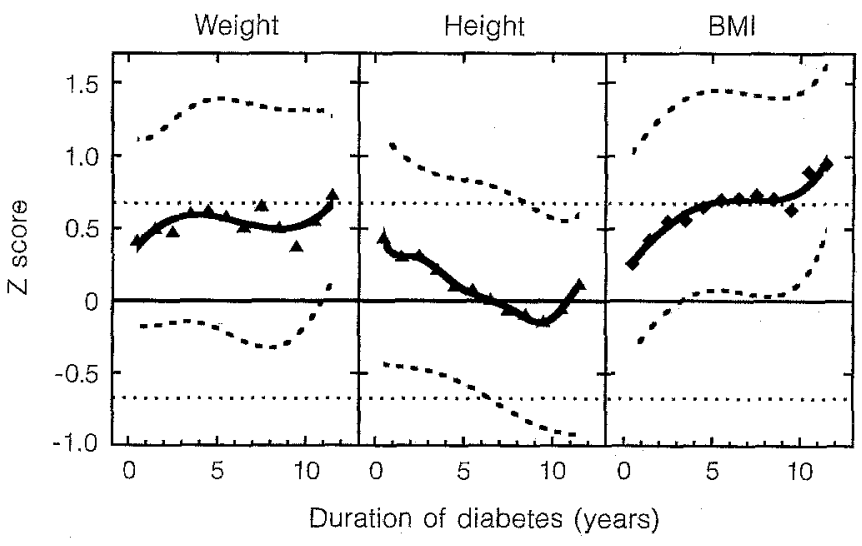

Fig. 1. Z scores for weight, height and BMI in 389 children with IDDM related to the duration of the disease. The symbols indicate the median ( $50^{\text {th }}$ percentile) for each yearly interval, the solid line represents a fitted spline curve for the median, while the dashed lines correspond to the $25^{\text {th }}$ and $75^{\text {th }}$ percentiles for the respective parameter. For comparison, the horizontal lines indicate the $50^{\text {th }}$ (solid) as well as $25^{\text {th }}$ and $75^{\text {th }}$ percentiles (dotted lines) for the reference population [13]. At the time of diagnosis, diabetic children were taller than average. This excess height was lost during the first 8 years of diabetes, but patients caught up during the subsequent years. In contrast, standardized weight remains above average, resulting in progressive overweight as reflected by BMI

\section{Results}

At the onset of IDDM, paediatric patients were significantly taller compared to the normative data of the Zurich longitudinal growth study (SD-scores: +0.37 $[-0.35-+1.08]$ for median, $25^{\text {th }}, 75^{\text {th }}$ percentiles, respectively; $p<0.001$ ). Initial height was not significantly different between boys and girls. During the subsequent course of diabetes the median $\mathrm{z}$ score for height progressively decreased, to $-0.15[-0.86-$ +0.57 ] after 9 years of diabetes (Fig. 1). After 10 years of diabetes, this height decrement was reversed: the group of diabetic children caught up again to $z$ scores for height above zero, as patients with 12 years of diabetes were on average taller than the reference population (SD-score + $0.11[-0.91-+0.59], n=72$ ).

Even during the first year of the disease, children with diabetes were heavier when compared to children from the Zurich growth study (z score for weight: $+0.41[-0.17-+1.11]$, for BMI: + 0.26 [-0.35-+ 1.03] (both $p<0.001$ ). During the first decade of diabetes, children became progressively overweight: $\mathrm{z}$ score for weight increased while height-SDS decreased, resulting in a considerable increment of BMI (Fig. 1). After 12 years of diabetes, median $\mathrm{z}$ score for BMI was $+1.23[+0.88+1.53]$ in boys and $+0.93[+0.44-$ $+1.58]$ in girls $(p<0.001$ vs control population, gender difference was not significant).

In order to clarify whether reduced longitudinal growth and progressive overweight occur at specific ages, weight, height and BMI of diabetic children were 


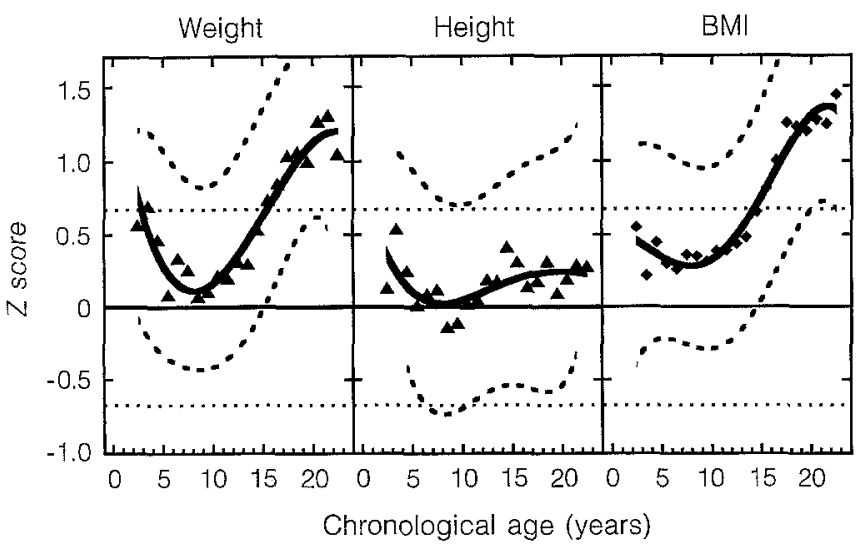

Fig. 2. Weight, height and BMI (z scores) in 389 children with IDDM related to chronological age. The format is identical with Figure 1. Obesity develops during and after puberty

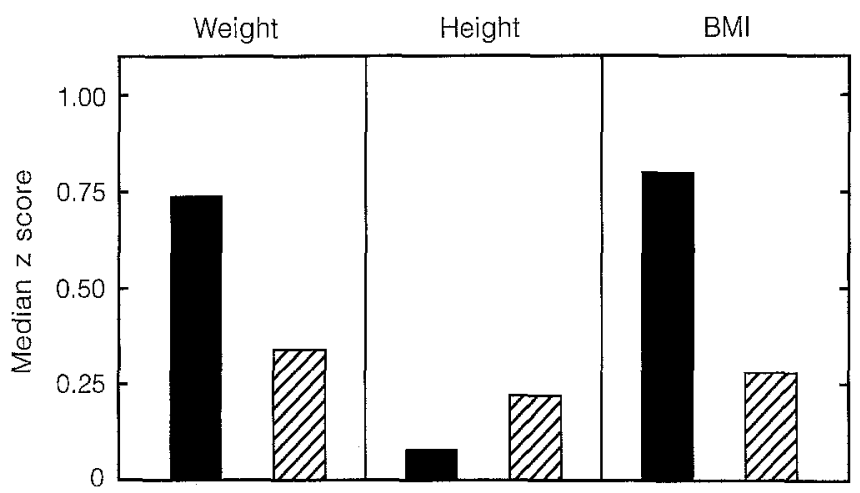

Fig.3. Z scores for weight, height and BMI in children with diabetes in comparison with the respective values in unaffected siblings $\square(n=186)$. Based on the most recent measurement, weight and BMI are increased significantly $(p<0.002$ and $p<0.0001$, respectively) in diabetic children (height, N.S.) (Wilcoxon test)

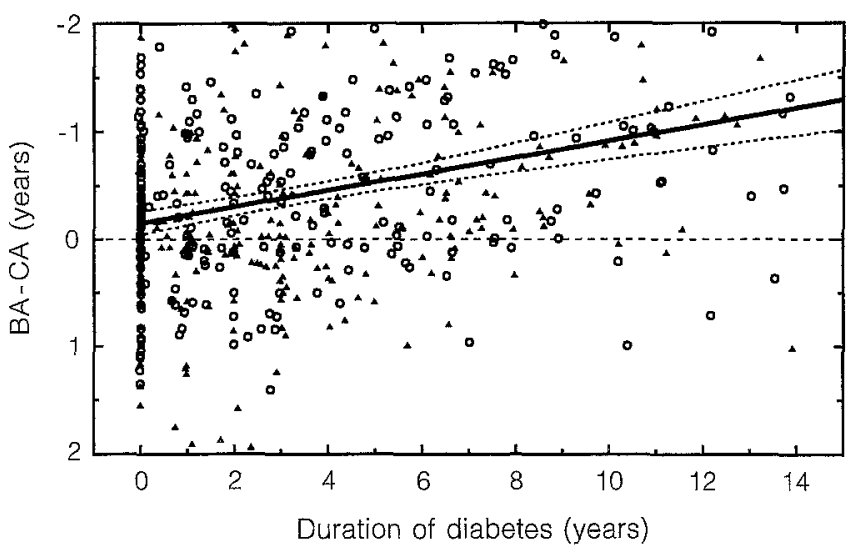

Fig. 4. Bone age retardation (bone age minus chronological age in years) in relation to the duration of diabetes. Symbols in the upper half of the figure represent patients with delayed bone ages, while in the lower half bone ages are advanced. A total of $514 \mathrm{X}$ rays from 294 children are included ( $\boldsymbol{\Delta}=$ boys, $\mathrm{O}=$ girls $)$. A significantly positive correlation between bone-age retardation and the duration of diabetes was present $(r=0.28$, $p<0.001$ ) related to their chronological age (Fig. 2). Standardized height was lowest in children 8-9 years of age, while both pre-school as well as pubertal and postpubertal children were taller compared to the Zurich reference data. Assuming adult height to be attained at a chronological age of 18 years, 76 patients were available for analysis. In this subgroup, SD score for height was $+0.30[-0.31-+1.11]$, nearly identical to the $\mathrm{z}$ score for height in siblings $(+0.22[-0.39-$ $+0.91]$ ), and not significantly below the age-adjusted height at diagnosis of diabetes $(\mathrm{z}$ score +0.37 , see above). It is evident from Figure 2, that overweight predominantly develops during the pubertal and postpubertal years. At the age of 18 years, $\mathrm{z}$ score for weight was $+1.06[+0.64-+1.75]$ and for BMI +1.23 $[+0.68-+1.92]$, with no significant differences between boys and girls.

In 186 unaffected siblings, the median $\left[25^{\text {th }}-75^{\text {th }}\right.$ percentile] of the SD score for height was +0.22 $[-0.39-+0.91]$,for weight $+0.34[-0.28-1.17]$, and $+0.28[-0.38-+1.14]$ for BMI (Fig. 3). These values were significantly above the reference data from the Zurich study in healthy children $(p<0.001)$. If the most recent measurements in children with diabetes were compared to the respective measurements in their unaffected siblings, the $\mathrm{z}$ score for height was not significantly different $(+0.08$ in diabetic compared to +0.22 in unaffected siblings). In contrast, weight and BMI were significantly elevated in affected children compared to their healthy siblings: (SDS for weight: $+0.74[+0.03-+1.47]$ in diabetic compared to +0.34 $[-0.28-+1.17]$ in unaffected siblings, $p<0.002$; SDS for BMI: $+0.80[+0.11-1.85]$ compared to +0.28 $[-0.38-+1.14], p<0.0001)$.

We next asked how parental height and weight is related to height and weight in diabetic children. Both mid-parental height [(father + mother)/2] as well as mid-parental weight were highly significantly related to the respective SD scores in their diabetic children $(r=+0.43, p<0.0001$ for height and $r=+0.23$, $p<0.002$ for weight). Identical coefficients of correlation were found between the parents and their unaffected children $(r=+0.43, p<0.0001$ for height and $r=+0.30, p<0.0001$ for weight). When boys and girls were evaluated separately, weight SD score was significantly correlated with mid-parental weight in unaffected boys $(r=+0.35, p<0.0006)$ as well as unaffected girls $(r=+0.26, p<0.02)$. Surprisingly, in diabetic girls, no such relationship was present: the coefficient of correlation with mid-parental weight was $r=+0.34, p<0.001$ for diabetic boys compared to $r=+0.14$, NS, in diabetic girls.

In order to test the hypothesis that the tempo of growth and development is retarded in diabetic children, 514 determinations of bone ages were evaluated in relation to chronological age and duration of diabetes (Fig. 4). At the onset of diabetes, bone age was not retarded (BA-CA: -0.125 years $[-0.64-+0.32]$, 
$n=110$ ). However, during the subsequent course of diabetes, bone ages were progressively delayed, resulting in a significantly positive correlation between bone age retardation and the duration or diabetes $(r=+0.28, p<0.001)$. No $\mathrm{X}$ rays for the determination of bone ages in non-diabetic siblings were available.

\section{Discussion}

Several studies have reported that at the onset of diabetes children are taller than expected $[2,6,7,9,14]$. However, normal [9] or even reduced height [15] at diagnosis of diabetes have also been reported, the latter finding in a study on identical twins followed up prospectively. In our study, including nearly 400 unselected patients from our hospital, height of both patients as well as their siblings fell above the reference data for height in healthy children. This finding is consistent with two interpretations: 1) the Zurich control group, originally consisting of more than 400 children born between 1950 and 1960, and followed up longitudinally for 20 years, may not be representative for children born in Southern Germany 15-20 years later [13]. To exclude this possibility, a large sample of height measurements in unaffected control children, representative for our area, would be necessary. Such data are currently not available. However, in our previous study, we included a control group of 102 healthy children [3]. Height and weight did not differ significantly from expected values. 2) In agreement with reports in the literature, increased height in diabetic children as well as their siblings is compatible with the notion that genetically determined tall stature and rapid growth are risk factors for the development of IDDM $[6,7]$.

In our study, age- and sex-standardized height in diabetic children was not significantly different from the respective measurements in their unaffected siblings. In addition, height was not significantly different between boys and girls. This agrees with several recent publications $[8,9,14]$, while other studies have reported boys with newly-diagnosed diabetes to be taller compared to girls [6,7].

During the early course of diabetes, diabetic children progressively lose height, and bone ages are increasingly retarded. Beyond 10 years of diabetes, children catch up again, and height in a subgroup of 76 patients followed up to final height (assumed with a chronological age of 18 years or more) was not reduced. These findings are compatible with the assumption of a transient retardation in growth and development during pre-pubertal and pubertal years. This agrees with previous publications on the early course of diabetes [3,16], although only few long-term follow-up studies are available so far. In the unselected group of all patients treated at our institution, short stature is not a relevant problem. However, subgroups of patients with insufficient metabolic control or coexistent undiagnosed celiac disease or hypothyroidism may not reach their genetic height potential.

Our data demonstrate significant and progressive overweight in paediatric patients with diabetes. Obese, but otherwise healthy children tend to be taller than average, have accelerated bone maturation and experience early puberty [17-19]. Therefore, in comparison, delayed growth and maturation in children with diabetes is even more remarkable. Overweight is significantly related to the development of hypertension in our diabetic patients [20] and represents a wellestablished risk factor for cardiovascular disease in diabetic patients [21]. In healthy children, overweight is associated with reduced pituitary secretion of $\mathrm{GH}$, while serum levels of $\mathrm{GH}$-binding protein are high and circulating concentrations of IGF-I are in the highnormal range [22]. In contrast, in diabetic subjects, GH secretion is elevated, while GH-BP and IGF-I are reduced [23-25]. These alterations in the GH-IGF-Iaxis provide further evidence that the hormonal regulation of longitudinal growth is altered in children suffering from diabetes.

It is likely that the degree of metabolic control is related to impaired longitudinal growth as well as the development of obesity in patients with diabetes. However, it is very difficult to accurately assess the quality of blood glucose control over long periods, as a considerable number of children included in this study developed diabetes before the advent of glycated haemoglobin as a routine long-term measurement of metabolic control, and the number of control visits naturally differs among patients. Not surprisingly, conflicting results have been published on the effect of glycaemic control on growth velocity in diabetic children $[4,5,16,26]$. In order to avoid a bias due to these problems, we did not attempt to relate anthropometric data to metabolic control in this study. A likely explanation for the development of overweight may be provided by recurrent hypoglycaemic episodes leading to additional calorie intake. The initiation of tight metabolic control in previously poorly-controlled diabetic patients is associated with weight gain, as recently demonstrated in the Diabetes Control and Complications Trial [27].

Our study demonstrates that growth and maturation in children with IDDM are slightly delayed, but adult height is not compromised. Genetic factors affect height to a similar degree in children both with and without diabetes. In contrast, additional factors specific for diabetes contribute to increased weight gain in diabetic children and adolescents. The development of overweight represents a major problem for the long-term outcome in diabetic patients. Future research will be directed towards identifying sub-populations at increased risk, as well as towards the devel- 
opment of strategies to maintain normal weight despite tight metabolic control in patients with IDDM.

Acknowledgements. We are indebted to H. Nebenführ, A. Thon, R. Völk and U.Weinstein for data collection, as well as to M. Gauss, J. Högel, S. Knäuer and F. Schweiggert for statistical and computational advice. This work was supported by a grant from the Federal Department of Health, Bonn, Germany ("Modellprojekt zur Verbesserung der Versorgung chronisch kranker Patienten"), and an institution-based grant from the State of Baden-Württemberg ("Landesschwerpunkt Diabetes").

\section{References}

1. Jackson RL (1984) Growth and maturation of children with insulin-dependent diabetes mellitus. Pediatr Clin North Am 31: 545-567

2. Salardi S, Tonioli S, Tassoni P, Tellarini M, Mazzanti I, Cacciari $\mathrm{E}$ (1987) Growth and growth factors in diabetes mellitus. Arch Dis Child 62: 57-62

3. Thon A, Heinze E, Feilen KD et al. (1992) Development of height and weight in children with diabetes mellitus: report on two prospective multicentre studies, one cross-sectional, one longitudinal. Eur J Pediatr 151:258-262

4. Wise JE, Kolb EL, Sauder SE (1992) Effect of glycemic control on growth velocity in children with IDDM. Diab Care 15: 826-830

5. Herber SM, Dunsmore IR (1988) Does control affect growth in diabetes mellitus? Acta Paediatr Scand 77: 303305

6. Drayer NM (1974) Height of diabetic children at onset of symptoms. Arch Dis Child 49: 616-620

7. Blom L, Persson LA, Dahlquist G (1992) A high linear growth is associated with an increased risk of childhood diabetes mellitus. Diabetologia 35: 528-533

8. Emmerson AJB, Savage DCL (1988) Height at diagnosis in diabetes. Eur J Pediatr 147: 319-320

9. Songer TJ, LaPorte RE, Tajima N et al. (1986) Height at diagnosis of insulin dependent diabetes in patients and their non-diabetic family members. BMJ 292: 1419-1422

10. Holl RW, Grabert M, Schweiggert F, Heinze E (1993) Ein Computerprogramm zur prospektiven Datenerfassung bei jugendlichen Patienten mit Typ I Diabetes mellitus. Arbeitserleichterung, Qualitätskontrolle und Verbesserung der Kommunikation zwischen Diabeteszentrum, Hausarzt und Patient. Diab Stoffw 3: 232-238

11. Heinze E, Holl RW (1990) Diabetes mellitus. In: Reinhardt D, von Harnack GA (eds) Therapy of pediatric diseases. $4^{\text {th }}$ Edition, Springer, Berlin Heidelberg New York, pp 83-92
12. Greulich WW, Pyle SI (1951) Radiographic atlas of skeletal development of the hand and wrist. Stanford Univ Press, Standford, CA

13. Prader A, Largo H, Molinari L, Issler C (1989) Physical growth of Swiss children from birth to 20 years of age. Helv Paediatr Acta [Suppl] 52: 1-125

14. Price DE, Burden AC (1992) Growth of children before onset of diabetes. Diabetes Care 15: 1393-1395

15. Leslie RDG, Lo S, Millward BA, Honour J, Pyke DA (1991) Decreased growth velocity before IDDM onset. Diabetes 40:211-216

16. Jivani SK, Rayner PHW (1973) Does control influence the growth of diabetic children? Arch Dis Child 48: 109-115

17. Vignolo M, Naselli A, DiBattista E, Mostert M, Aicardi D (1988) Growth and development in simple obesity. Eur J Pediatr 147: 242-244

18. Frisch RE (1985) Fatness, menarche, and female fertility. Perspect Biol Med 28: 611-633

19. Lloyd JK, Wolff OH, Whelen WS (1961) Childhood obesity: a long-term study of height and weight. BMJ 1: 145-148

20. Holl RW, Rehm R, Walker P, Heinze E (1992) 24-h blood pressure recording in children and adolescents with type I diabetes mellitus: effects of duration of diabetes, overweight and urinary albumin excretion. Z Kardiol 81 (S2): 25-27

21. Donahue RP, Orchard TJ (1992) Diabetes mellitus and macrovascular complications. Diabetes Care 15: 1141-1155

22. Holl RW, Snehotta R, Siegler B, Scherbaum W, Heinze E (1991) Binding protein for human growth hormone: effects of age and weight. Horm Res 35: 190-197

23. Edge JA, Dunger DB, Matthews DR, Gilbert JP, Smith CP (1990) Increased overnight growth hormone concentrations in diabetic compared with normal adolescents. J Clin Endocrinol Metab 71: 1356-1362

24. Holl RW, Siegler B, Scherbaum WA, Heinze E (1993) The serum growth hormone-binding protein is reduced in young patients with insulin-dependent diabetes mellitus. J Clin Endocrinol Metab 76: 165-167

25. Lanes R, Reckert B, Fort P, Lifshitz F (1985) Impaired somatomedin generation test in children with insulin-dependent diabetes mellitus. Diabetes 34: 156-160

26. Clarson C, Daneman D, Ehrlich RM (1985) The relationship of metabolic control to growth and pubertal development in children with insulin-dependent diabetes. Diabetes Res 2: 237-241

27. The Diabetes Control and Complications Trial Research Group (1993) The effect of intensive treatment of diabetes on the development and progression of long-term complications in insulin-dependent diabetes mellitus. $\mathrm{N}$ Engl J Med 329: 977-986 